CDF Note 7738

July 5, 2005

\title{
SEARCH FOR SUPERSYMMETRY AT THE TEVATRON DIS'05, WISCONSIN, USA
}

\author{
John Zhou \\ on behalf of CDF and D $\varnothing$
}

\begin{abstract}
We report at the DIS'05 conference the latest results of search for supersymmetry (SUSY) at the Fermilab Tevatron. No evidence of Supersymmetry is found and limits are set accordingly.
\end{abstract}




\title{
Search for Supersymmetry at the Tevatron DIS'05, Wisconsin, USA
}

\author{
John Zhou \\ on behalf of the CDF and $\mathrm{D} \emptyset$ collaborations \\ P.O.Box 500 \\ MS 318 (Rutgers University) \\ Fermi National Accelerator Laboratory \\ Batavia, IL 60510
}

\begin{abstract}
We report at the DIS'05 conference the latest results of search for supersymmetry (SUSY) at the Fermilab Tevatron. No evidence of Supersymmetry is found and limits are set accordingly.
\end{abstract}

Keywords: Supersymmetry, Tevatron, Run2, CDF, DØ

PACS: $12.60 . \mathrm{Jv}$

\section{Introduction}

Tevatron Run2 has been taking data smoothly since 2003 . The analyses reported here are based on $200-390 \mathrm{pb}^{-1}$ of CDF and D $\varnothing$ data. They include results of Supersymmetry searches for R-parity conserving and violating scenarios under the framework of minimal supergravity (mSUGRA), gauge mediated SUSY breaking (GMSB), or anomaly mediated SUSY breaking (AMSB).

\section{Search for $\widetilde{\chi}_{1}^{ \pm} \widetilde{\chi}_{2}^{0}$ Pair Production via the Tri-lepton Final States}

The chargino-neutralino $\left(\widetilde{\chi}_{1}^{ \pm} \widetilde{\chi}_{2}^{0}\right)$ pair decaying to 3 isolated leptons is consider the "golden" channel for SUSY search because there is little standard model (SM) background which is dominated by fakes and $W \gamma$. For the results in this section, the underlying framework is mSUGRA.

DØ carried out 6 analyses based on the final states listed in Table 1. After all the optimized cuts and a $p_{T}^{l 3}>4 \mathrm{GeV}$ cut on the third lepton are made, 4 data events are observed in $320 \mathrm{pb}^{-1}$ of data with $3.85 \pm 0.75$ expected. Since no SUSY signal is observed, the results of the six channels are combined to set limits on the mass of the chargino: $m_{\widetilde{\chi}_{1}^{ \pm}}>117$ (132) GeV/c $c^{2}$ for the 3l-max (heavy-squarks) scenario at the 95\% confidence level. The leptonic branching fraction is maximally enhanced in the $3 l$-max scenario and the scalar mass unification is relaxed in the heavy-squarks scenario. These limits are beyond those by LEP although the LEP limits are model independent. 
TABLE 1. Left: Number of expected background and observed events in $320 \mathrm{pb}^{-1}$ of data in the six individual DØ tri-lepton analyses and in total. Right: The 95\% confidence limits on $\sigma \times B R$ as a function of chargino mass.

\begin{tabular}{ccc} 
Channel & Expected & Observed \\
\hline$e e+l$ & $0.21 \pm 0.12$ & 0 \\
$e \mu+l$ & $0.31 \pm 0.13$ & 0 \\
$\mu \mu+l$ & $1.75 \pm 0.57$ & 2 \\
$\mu^{ \pm} \mu^{ \pm}$ & $0.64 \pm 0.38$ & 1 \\
$e \tau+l$ & $0.58 \pm 0.14$ & 0 \\
$\mu \tau+l$ & $0.36 \pm 0.11$ & 1 \\
All & $3.85 \pm 0.75$ & 4 \\
\hline
\end{tabular}

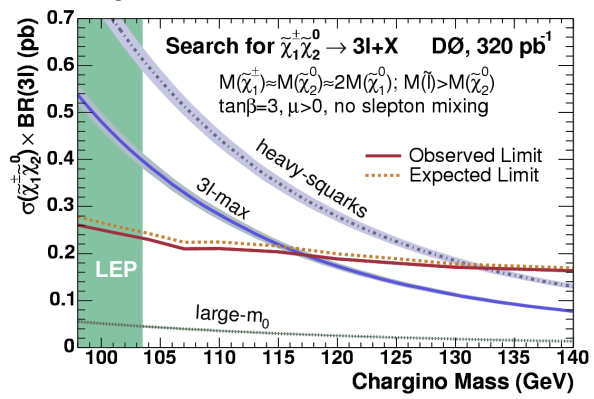

CDF also completed two analyses in the high and low $p_{T} e e+l$ channel using data from different electron triggers. The low $p_{T}$ analysis includes hadronic $\tau$ contribution by allowing just an isolated track in the detector in addition to the two electrons. The luminosity used, the number of expected and observed data events are listed in Table. 2. Results in the $\mu \mu+l$ and $e \mu+l$ channels, and all channels combined are expected soon.

TABLE 2. Number of expected signal, background and observed events in 346 and $224 \mathrm{pb}^{-1}$ of data in the two individual CDF tri-lepton analyses. The signal is mSUGRA with $m_{0}=100 \mathrm{GeV}, m_{1 / 2}=180 \mathrm{GeV}, \tan \beta=5, \mu>0$, and $A_{0}=0$.

\begin{tabular}{ccccc} 
Channel & Luminosity & Expected Signal & Expected Background & Observed \\
\hline$e e+l$ high $p_{T}$ & $346 \mathrm{pb}^{-1}$ & 0.5 & $0.16 \pm 0.07$ & 0 \\
$e e+l$ low $p_{T}$ & $224 \mathrm{pb}^{-1}$ & 0.5 & $0.36 \pm 0.27$ & 0 \\
\hline
\end{tabular}

\section{Search for $\widetilde{\chi}_{1}^{ \pm} \widetilde{\chi}_{2}^{0}$ Pair Production via the Diphoton Final States}

In the GMSB model, the gravitino can be the lightest SUSY particle and the neutralino, $\widetilde{\chi}_{1}^{0}$, can be the next lightest SUSY particle. A $\widetilde{\chi}_{1}^{ \pm} \widetilde{\chi}_{2}^{0}$ pair production may result in diphoton $+E_{T}$ final state with $\widetilde{\chi}_{1}^{0}$ decaying to a photon and a gravitino. CDF and DØ searched in this final state in 200 and $263 \mathrm{pb}^{-1}$ of data, respectively. The analysis cuts, background expectation, and observation are listed in Table. 3. Background is dominated by $e \gamma$ and non-collision events. Combining the CDF and D $\varnothing$ results, we set a limit: $m_{\widetilde{\chi}_{1}^{ \pm}}>209 \mathrm{GeV} / c^{2}$ at the $95 \%$ confidence level as shown in the left plot in Fig. 1 .

TABLE 3. Number of expected SM background and observed events in 263 and $202 \mathrm{pb}^{-1}$ of data in the $\mathrm{D} \emptyset$ and CDF diphoton $+E_{T}$ analyses.

\begin{tabular}{cccccc} 
Experiment & Luminosity & $E_{T}^{\gamma}$ cut & $E_{T}$ cut & Expected Background & Observed \\
\hline $\mathrm{D} \emptyset$ & $263 \mathrm{pb}^{-1}$ & $20 \mathrm{GeV}$ & $45 \mathrm{GeV}$ & $3.7 \pm 0.6$ & 2 \\
$\mathrm{CDF}$ & $202 \mathrm{pb}^{-1}$ & $13 \mathrm{GeV}$ & $40 \mathrm{GeV}$ & $0.3 \pm 0.1$ & 0 \\
\hline
\end{tabular}




\section{Search of Gluino-Squark in Hadronic Final States}

If they exist, squarks $(\widetilde{q})$ and gluinos $(\widetilde{g})$ are copiously produced at the Tevatron because they couple strong to gluons and quarks. The typical signatures are hard jets and large $E_{T}$ resulted from chain decays of squarks and/or gluinos. The largest background come from QCD multijet events in which the jet energies are mis-measured giving rise to large $E_{T}$.

DØ divided the final states into 2,3 , and 4 jets $+E_{T}$, each optimized for $\widetilde{q} \widetilde{q}, \widetilde{q} \widetilde{g}$, and $\widetilde{g} \widetilde{g}$ pair production, respectively. The cuts on jet $p_{T}$, event $H_{T}=\sum p_{T}^{\mathrm{jet}}$, and $E_{T}$, and the resulting expected background and observed number of events based on $310 \mathrm{pb}^{-1}$ of data are listed in Table. 4. Since no evidence of squark or gluino production is found, a limit is set as a function of squark and gluino mass as shown in the middle plot in Fig. 1.

TABLE 4. Left: Cuts on the number of jets, $p_{T}$, event $H_{T}$, and $E_{T}$; the resulting expected number of background events and observed number of events in $310 \mathrm{pb}^{-1}$ of data for the $\mathrm{D} \emptyset$ analyses to search for squarks and gluinos.

\begin{tabular}{ccccc} 
\# jets $\left(p_{T}(\mathrm{GeV})\right)$ & $H_{T}(\mathrm{GeV})$ & $E_{T}(\mathrm{GeV})$ & Expected & Obs. \\
\hline 2 jets $(60,50)$ & 250 & 175 & $12.8 \pm 5.4$ & 12 \\
3 jets $(60,40,25)$ & 325 & 100 & $6.1 \pm 3.1$ & 5 \\
4 jets $(60,40,30,25)$ & 175 & 75 & $9.3 \pm 0.5$ & 10 \\
\hline
\end{tabular}

CDF also performed a similar analysis in a 3 jet $+E_{T}$ channel. The 3 jets are required to have $E_{T}>125,75$, and $25 \mathrm{GeV}$, respectively. In addition, the event must pass the cuts: $H_{T}=E_{T}^{j 1}+E_{T}^{j 2}+E_{T}^{j 3}>350 \mathrm{GeV}$ and $E_{T}>165 \mathrm{GeV} .4 .2 \pm 1.1$ background events are expected with 3 events observed in $254 \mathrm{pb}^{-1}$ of data. A limit on the masses of gluino and squark is being set.

\section{Search for SUSY in the R-parity violating processes}

DØ searched for slepton in the mSUGRA framework in four different channels. The coupling vertices, the corresponding final states considered, and the results are shown in Table. 5. In each analysis, only the coupling under investigation is assumed to dominate.

TABLE 5. Left: R-parity violating slepton search results. Right: the limit contour resulted from the $\mu \mu+2$ jet analysis on the $\lambda_{211}^{\prime}$ coupling.

\begin{tabular}{ccccc} 
Coupling & $\lambda_{211}^{\prime}$ & $\lambda_{121}$ & $\lambda_{122}$ & $\lambda_{133}$ \\
\hline$\mu$ & $<0$ & $>0$ & $>0$ & $>0$ \\
$\tan \beta$ & 2 & 5 & 5 & 10 \\
Channel & $\mu \mu+2$ jets & $e e l+E_{T}$ & $\mu \mu l+E_{T}$ & $e e \tau+E_{T}$ \\
Lum $\left(\mathrm{pb}^{-1}\right)$ & 154 & 238 & 160 & 199 \\
$\#$ Expected & $1.1 \pm 0.4$ & $0.5 \pm 0.4$ & $0.6 \pm 1.9$ & $1.0 \pm 1.4$ \\
$\#$ Observed & 2 & 0 & 2 & 0 \\
$m_{\widetilde{\chi}_{1}^{0}\left(\mathrm{GeV} / c^{2}\right)}$ & Fig. & $>95$ & $>90$ & $>66$ \\
$m_{\widetilde{\chi}_{1}^{ \pm}}\left(\mathrm{GeV} / c^{2}\right)$ & Fig. & $>181$ & $>165$ & $>118$ \\
\hline
\end{tabular}

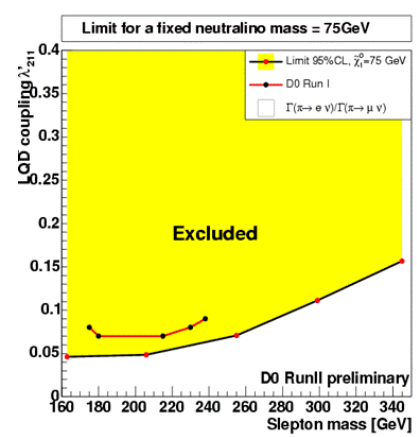


Using $200 \mathrm{pb}^{-1}$ of data CDF investigated the coupling $\lambda_{333}^{\prime}$ in the process of stop pair production and decaying into $2 \tau$ leptons and $2 \mathrm{~b}$-jets. The mSUGRA framework and $B R\left(t_{1} \rightarrow b \tau\right)=100 \%$ are assumed. It is required that one $\tau$ decays hadronically and the other decays leptonically. There is no b-tagging required in order to obtain maximal acceptances. After all optimal cuts we expect $4.8 \pm 0.7$ events and observe 5 . From that we are able to extract a limit on $m_{t_{1}}>129 \mathrm{GeV} / c^{2}$, as shown in the right plot in Fig. 1.

\section{Search for Charged Mass Particles (CHAMP) at DØ}

Charginos in the AMSB models or the $\widetilde{\tau}$ in the GMSB models may be long lived and decay outside of the detector $(c \tau>10 \mathrm{~m})$. They leave their trace as minimum-ionizing particles moving at a speed much less than the speed of light in the detector. Using $390 \mathrm{pb}^{-1}$ of data, DØ searched for the pair production of CHAMPs in the dimuon channel. The muons are required to have $p_{T}>15 \mathrm{GeV}$ and that they must have a large speed significance defined as: $S_{v}=(1-$ speed $) / \sigma_{\text {speed }}$. After optimization for the signal in a $2 \mathrm{D}$ plane in di-muon mass and $S_{v}^{1} \times S_{v}^{2}, 0.66 \pm 0.06$ background events are expected and 0 is observed. A limit is set on the chargino masses in the framework of AMSB: 140 (174) $\mathrm{GeV} / \mathrm{c}^{2}$ if the chargino is higgsino (gaugino) like.
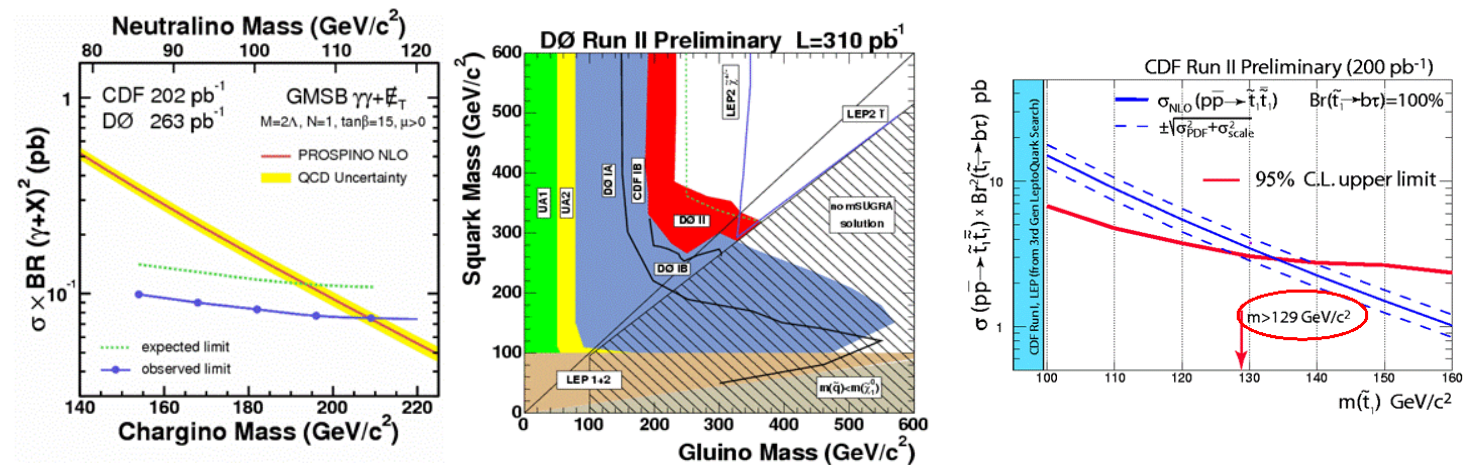

FIGURE 1. Left: combined CDF/D $\emptyset$ limit on $m_{\widetilde{\chi_{1}^{ \pm}}}$in GMSB model in the diphoton $+E_{T}$ analyses. Middle: limit as a function of masses of squark and gluino from DØś jets $+E_{T}$ analysis. Right: limit on $m_{\tilde{t}}$ from CDF di- $\tau+$ di-jet analysis.

\section{Conclusion}

With no SUSY signal observed, limits on the SUSY model parameters have been placed. Some limits, for example, the masses of the gluino and squarks are already beyond the world's current limit. With delivered luminosity just surpassing $1 \mathrm{fb}^{-1}$, new limits and even discoveries will become a reality soon. References to the results reported here as well as the latest results can be obtained at http://www-cdf.fnal.gov/physics/exotic/exotic.html and http://wWw-d0.fnal.gov/Run2Physics/WWW/results/np.html. 